\title{
Measurement Reliability for Acupoint Activity Determined with the Prognos Ohmmeter
}

\author{
L. Turner $\cdot$ W. Linden $\cdot$ A. Talbot Ellis • \\ R. Millman
}

Published online: 20 January 2010

(c) The Author(s) 2010. This article is published with open access at Springerlink.com

\begin{abstract}
The concepts of meridians and acupoints are critical to traditional Chinese medicine but are met with skepticism in Western medicine. Empirical validation of these concepts is in its beginning stages and still hampered by problems with measurement. A promising avenue and foundation for validity testing is the demonstration that acupoint activity can be reliably measured via determination of electrical resistance at well-defined body surface points. In this article, efforts are described to maximize measurement reliability; we tested a variety of protocols to determine which method of data aggregation is associated with maximal reliability. Twenty-one healthy individuals were subjected to 5 repeated measurement cycles to test the predicted increase in reliability with increasing number of aggregated measurements. Reliability, defined as internal consistency, was indeed highest for 5 measurements (mean alpha $=.88)$. Even the aggregate of only three measures was quite reliable (alpha $=.84$ ). Reliability for measuring acupoints on the left side of the body was roughly .05 higher than on the right side. Consistent with previous literature, we conclude that with repeated measures the reliability of electrical resistance measurements at acupoints is high and that a strong foundation for validation research is now laid.
\end{abstract}

Keywords Acupoints · Reliability $\cdot$ Measurement

L. Turner $(\bowtie) \cdot$ W. Linden $\cdot$ A. Talbot Ellis $\cdot$ R. Millman Department of Psychology, The University of British Columbia, Vancouver, BC, Canada

e-mail: 1turner@langara.bc.ca

\section{Introduction}

Western medicine has an uneasy relationship with Traditional Chinese Medicine (TCM). One reason for this 'disconnect' is that TCM is a blend of philosophy of human functioning and medical practice. It does not use aggressive interventions and is geared, in good part, toward prevention. Furthermore, TCM does not place the same emphasis as Western Medicine on quantification and randomized controlled trial methodologies. On the other hand, it would be hard to accept the notion that medical practices applied in the world's most populous country for hundreds of years have no substance or effects. Among the particularly contentious topics is that of the existence of meridians that connect defined body surface areas with specific functions and inner organs. Nevertheless, there is growing acceptance that the claimed surface points of meridians correspond with acupoints that, in turn, are routinely targeted in acupuncture.

TCM is based upon the meridian and acupoint system. In TCM, energy labeled 'Qi' is thought to flow through the meridians in a balanced and harmonious way. The meridian system is seen as a communication link between the surface of the body and the internal organs and provides a communication network to transport this vital energy or Qi (Yung 2004). The acupoints are distributed along the meridian channels (Yung 2004). In illness states, the Qi presumably becomes blocked at one or more acupoints. When an acupuncturist manipulates the acupoint site with needles, massages it, or applies electrical stimulation then the flow of Qi is restored.

The meridian system is analogous to the nervous system but is posited to be a functional descriptor and not necessarily an anatomical structure. Nevertheless, Voll (1978) first demonstrated that there are numerous locations over 
the body that have lower electrical resistance and greater conductivity than the surrounding tissue. When these points are mapped out they lie along the channels described in TCM as the meridian system (Yung 2004). Electrical impedance values have been determined to be approximately $0.8 \mathrm{MOhms}$ at the acupoints and $1.4 \mathrm{MOhms}$ at the surrounding tissue (Yung 2004). Other researchers have also confirmed that electrical resistance at acupoints was significantly lower than resistance at control points (Zhang et al. 2004). Furthermore, using sophisticated laser Doppler flowmetry, Hsin and colleagues (2007) demonstrated that acupoints have a significantly increased blood supply in the micro- vascular bed compared to that found in surrounding tissues. Lo (2002) was able to differentiate the acupoint system from surrounding tissue using the optical methods of the biophoton technique and infrared diffuse reflectance spectroscopy. While other research has demonstrated that light of certain wavelengths propagates more easily along the pericardium meridian compared to the non-meridian path and that the optical properties of meridians are significantly different from those of the surrounding tissue (Yang et al. 2007). Together, these studies support that meridians possess concurrent validity with functional biological measures that are commonplace in Western Medicine.

If acupoint activity can be reliably measured, then this knowledge can also support further research on acupuncture outcomes. Acupuncture is the most widely practiced subtype of TCM and a good-sized body of research has investigated outcomes. In acupuncture, the practitioner uses fine-tipped needles to activate electrical activity at specific body sites, namely the acupoints. The pathways through which acupuncture modifies physiological function to relieve pain and provide symptom relief are not fully understood; a suggested mechanism is that acupuncture triggers endorphin release. A recent review by Ahn and colleagues (2008) included nine studies that explored acupuncture sites and nine studies that explored the existence of a meridian system. They indicated that five out of nine studies showed a positive association between lower electrical resistance and acupuncture points, and seven out of nine studies showed a positive association between acupuncture meridians and lower electrical impedance and higher capacitance. They caution, however that the quality of the research in the area is poor and that the evidence that acupuncture points and meridians are electrically distinguishable is not conclusive. On the other hand, they do conclude that the preliminary findings are consistent and support further research in this area.

Several Cochrane Reviews have been conducted to evaluate the efficacy of acupuncture. A review of 26 trials, including 1,151 patients, demonstrated acupuncture to be effective in the treatment of idiopathic headache (Melchart et al. 2001). However, measurement and trial methodology was seen as of mixed quality. Similarly, electro-acupuncture was reported as effective in reducing chemotherapy induced nausea and vomiting (Ezzo et al. 2006) based on a review of 11 trials with 1,247 patients. Based on the results from 35 randomized controlled trials, Furlan et al. (1999) concluded that acupuncture was more effective for pain relief and functional improvement than no treatment or sham treatment for chronic back pain. However, this benefit waned with time.

\section{Rationale for the Current Study}

In order to advance basic research on acupoints, support effectiveness trials of acupuncture, and possibly other forms of alternative medicine practice, methods need to be available that build a bridge between TCM and Western Medicine. Among innovative methods trying to capture and quantify the flow of Qi energy is the determination of electrical resistance that provides 24 readings in ohms (one per acupoint) per measurement cycle (12 acupoints on each side of the body).

Arguably the best known such device is the Prognos (Med Prevent, Waldershof, Germany). Colbert and colleagues (2004) have tested the reliability of the Prognos device in 31 healthy participants, 17 females and 14 males, age range 24-63 years. Two hundred and eighty-eight measurements were subjected to statistical analysis. All subjects completed three trials. The mean reliability for trial one was .76 with a range of .54-.88. When the acupoint was marked with nontoxic washable ink and re-measured, the mean reliability of a single measurement increased to .85 (range .69-.96). The highest mean reliability of .96 (range .89-.99) was made in trial 3 when four measurements were made in rapid succession. The authors report that higher reliability correlated with lower mean electrical skin resistance. They also suggest that the reliability of acupoint measurement on the right side of the body (considered "yang" or male in Chinese medicine) was lower than measurements on the left side of the body (considered "yin" or female in Chinese medicine) (Colbert et al. 2004).

To date there is some early work on criterion validation for the ability of skin surface resistance measures to differentiate disease states. Szopinski et al. (2004) measured electrical impedance at 90 auricular organ projection areas in 180 subjects using a machine similar to the Prognos. Ninety of these subjects had medically confirmed disease conditions and 90 were healthy. These researchers were able to differentiate between healthy and disease states by the electrical resistance detected at auricular organ projection sites within the meridian system (Szopinski et al. 1998, 2004, 2006). 
We posit that the addition of validation research on acupoints may be of limited scientific value if there is doubt in the reliability of the underlying measurement methodologies. The first studies done so far are promising in that reliability coefficients are high and in the range of other widely used biomedical assessments like blood pressure where reliability coefficients are also in the .8-.9 range (Colbert et al. 2004; Pearson et al. 2007; Selenta et al. 2000). Given that the two previous reliability studies on acupoint measurement came from the same laboratory, it will strengthen the results of the Colbert group if independent corroboration can be obtained. If a high degree of consistency exists across settings, it may then be time to suggest a standardized measurement protocol for all future studies on acupoint activity.

\section{Study Objective}

As part of a larger project on the validation of the Prognos system, tapping into its ability to distinguish chronic pain from age-matched non-pain patients, we conducted pilot work on the tool's reliability. We also wanted to test empirically how many readings should be averaged to maximize test reliability given the established knowledge that a test's internal consistency and test-retest reliability improve with multiple measurements.

\section{Method}

\section{Instruments}

The Prognos Machine was obtained from the manufacturer (Med Prevent) and was calibrated by the company in Germany. The Prognos is an ohmmeter consisting of a power source connected by a cable to the measuring probe and a reference electrode $(6 \times 3.5 \mathrm{~cm})$ that is attached with a Velcro strap to the surface of the forearm. Impedance measurements are recorded as direct current analogue values taken while holding the probe at a 90 degree angle to the acupoint location. The analogue values are fed into an amplifier in the Prognos and transformed into a digital value by an $\mathrm{A} / \mathrm{D}$ converter. A digital screen displays the measured resistance values in kilo-ohms and the data are imported into a laptop computer via a serial cable (software by MedPrevent, Waldershof, Germany).

The Prognos instrument utilizes a $4.57 \mathrm{~mm}$ diameter flexible spring loaded probe tip and calculates an average electrical skin resistance value from 400 measurements taken in approximately $200 \mathrm{~ms}$ (www.medprevent.com). The probe tip, has a maximum excursion of $6.91 \mathrm{~mm}$, is connected to a linear spring and lies flush to the plastic

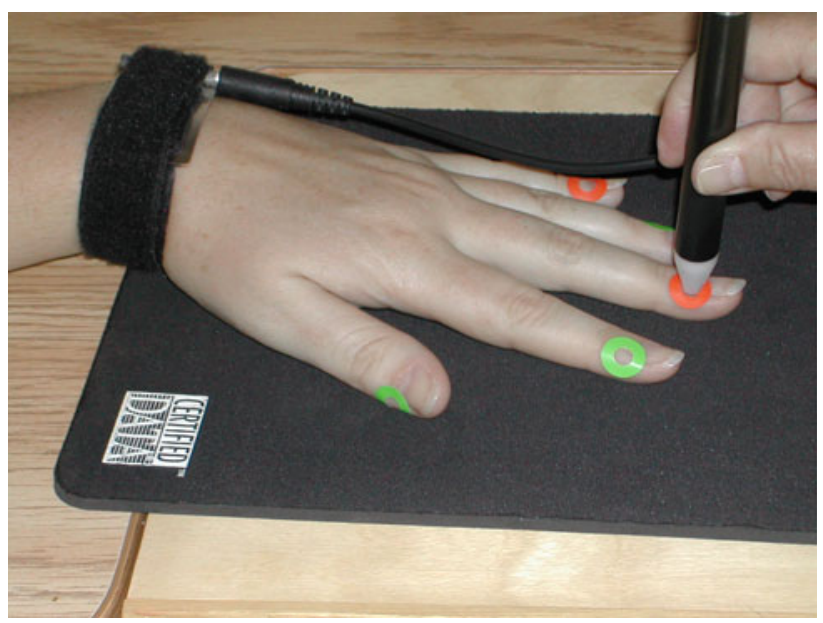

Fig. 1 Measurement with prognos probe

insulation at the end of a plastic cylinder (see Fig. 1). Within the cylinder a light emitting diode transmits a light beam to a photo detector and the spring loaded probe disrupts the light beam and triggers a reading at an average deflection of $2.90 \mathrm{~mm}$ with an average force of $2.68 \pm 0.05$ Newton (Colbert et al. 2004). When triggered, the Prognos applies 1.1 mille Amperes of current from the lower forearm strap to the probe tip for an average of $223 \pm 3 \mathrm{~ms}$.

\section{Procedures}

All measurements took place at the University of British Columbia. Like many physiological functions, electrical skin resistance at acupoints demonstrates a $24 \mathrm{~h}$ pattern of physiological variability. The values of electrical skin resistance increase dramatically and significantly during sleep and decrease upon awakening (Colbert et al. 2006) thus requiring some degree of experimental control if resistance values are to be compared across patients. Testing took place between the hours of 8:00 AM and 12:00 PM. Ambient temperature during testing ranged from $19-22^{\circ} \mathrm{C}$.

The data were collected by the first author as experimenter and a research assistant. The experimenter had been using the device for 9 months and had received training by staff from Med Prevent (Hammerschlag, Germany). The research assistant was trained by the experimenter in the use of the Prognos over a two day observation and practice session.

Participants were asked to sit quietly in an upright reclining chair. When data were collected from the toes, the reclining chair was activated so that the feet were elevated. Participants were told that the data were to be used to test the reliability of the instrument and that the measurements would be repeated 5 times in rapid 
succession. At the end of the measurement period participants were shown the graphical depiction of their meridian system and any questions pertaining to their results were answered.

Despite attempts to follow the methods described by Colbert et al. (2004), we had initial difficulties in obtaining reliable readings across different measurements. This led us to experiment with systematic strategies to make the measurement process quicker and more reliable. Colbert et al. (2004) suggest that marking the Jing well (Ting) meridian points increases the measurement reliability. However, through discussion with Med Prevent in Germany we learned that the use of a ball point pen or other form of ink marking may alter the resistance at the skin surface. Colbert (personal communication, April, 2009) suggested that we use adhesive rings used for magnetic acupoint therapy. We tried this but found that the flesh colour of the rings were not that helpful in locating the actual point. Instead, we tried coloured reinforcers for 3hole punch note paper. These seemed to work well. Research assistants placed the reinforcers on the fingers and toes of each participant at each of the 24 Jing well (Ting) acupoint locations. This ensured the correct placement of the Prognos probe with each measurement and saved time (see Fig. 1).

The reference electrode was secured to the left wrist with a Velcro strap. All measurements were taken with the subject sitting in the reclining chair. Participants were not able to see the computer screen as measurements were taken. Electrical resistance at the 24 Jing well (Ting) acupoints was recorded during 5 rapid repeat measurement cycles. Once a participant was prepared and the marker rings were placed over the acupoints, each complete measurement cycle took about $2 \mathrm{~min}$.

\section{Participants}

A sample of convenience was used for this study. Twentyone healthy subjects ranging from 23 to 64 years of age volunteered to participate in this study. There were 3 males and 18 females who participated. Participants were members of a class from a local College in Vancouver.
Table 1 Reliability of acupoint readings as a function of measurement repetitions
Overall mean $.81, .84, .85, .88$, .86

Mean left side $.84, .84, .88, .90$, .88

Mean right side $.78, .83, .83$, $.86, .83$

Reliability Coefficients (Cronbach's alpha) for each Meridian as a function of the number of measurements that were averaged)

\begin{tabular}{|c|c|c|c|c|c|}
\hline \multicolumn{6}{|c|}{ Number of measurements } \\
\hline Meridian & 2 & 3 & 4 & 5 & $\begin{array}{l}\text { The last } 4 \text {, } \\
\text { eliminating } \\
\text { the first reading }\end{array}$ \\
\hline Lung-L & .87 & .93 & .93 & .95 & .94 \\
\hline Lung-R & .88 & .89 & .90 & .91 & .91 \\
\hline Large Intestine-L & .84 & .92 & .93 & .96 & .94 \\
\hline Large Intestine-R & .77 & .87 & .90 & .88 & .84 \\
\hline Stomach-L & .88 & .84 & .85 & .90 & .88 \\
\hline Stomach-R & .65 & .79 & .85 & .87 & .88 \\
\hline Spleen Pancreas-L & .87 & .85 & .88 & .89 & .87 \\
\hline Spleen Pancreas-R & .67 & .80 & .80 & .85 & .82 \\
\hline Heart-L & .75 & .80 & .88 & .84 & .84 \\
\hline Heart-R & .85 & .88 & .92 & .91 & .86 \\
\hline Small Intestine-L & .80 & .82 & .88 & .91 & .90 \\
\hline Small Intestine-R & .93 & .85 & .91 & .93 & .91 \\
\hline Bladder-L & .90 & .86 & .88 & .87 & .79 \\
\hline Bladder-R & .59 & .72 & .85 & .87 & .82 \\
\hline Kidney-L & .91 & .92 & .92 & .96 & .94 \\
\hline Kidney-R & .88 & .83 & .86 & .90 & .87 \\
\hline Circulation-L & .83 & .69 & .76 & .81 & .74 \\
\hline Circulation-R & .75 & .89 & .92 & .91 & .91 \\
\hline Triple Heater-L & .77 & .82 & .90 & .92 & .94 \\
\hline Triple Heater-R & .79 & .74 & .33 & .47 & .42 \\
\hline Gall Bladder-L & .80 & .80 & .84 & .92 & .93 \\
\hline Gall Bladder-R & .87 & .86 & .87 & .89 & .89 \\
\hline Liver-L & .88 & .88 & .87 & .89 & .85 \\
\hline Liver-R & .74 & .81 & .86 & .88 & .86 \\
\hline
\end{tabular}




\section{Results}

The mean reliabilities for various forms of data aggregation for 5 consecutive trials were computed. Inspection of the reliability coefficients for all 24 acupoints revealed an overall very consistent pattern of reasonably good reliability even with only 2 measures (above a threshold of alpha $=.80)$ and then steady improvements in scores as the aggregation increased to 5 readings (.88; see Table 1$)$. At the maximum of 5 aggregated scores the range of observed Cronbach's alpha values for each of the 24 acupoints was .47-.96 (for descriptives of each of the acupoints see Table 2). However, the coefficient of .47 pertained to the Right Triple Heater acupoint and when excluding this outlier score, the range of Cronbach's alphas for all remaining 23 acupoints was .84-.95. The Left Triple Heater acupoint did not show this same trend toward low reliability.

We also assessed whether reliability increased when the first reading was excluded from the averaged readings as it may be particularly unstable due to novelty for the participant. This exclusion did not serve to improve reliability (see Table 1).

Table 2 Means and standard deviations of acupoints

\begin{tabular}{|c|c|c|}
\hline Meridian & $M$ & $S D$ \\
\hline Lung-L & 9442.10 & 10750.88 \\
\hline Lung-R & 8313.19 & 8281.29 \\
\hline Large Intestine-L & 10087.36 & 10888.56 \\
\hline Large Intestine- $\mathrm{R}$ & 6396.40 & 5731.60 \\
\hline Stomach-L & 3997.20 & 2178.31 \\
\hline Stomach-R & 4442.50 & 2287.85 \\
\hline Spleen Pancreas-L & 5823.17 & 4003.00 \\
\hline Spleen Pancreas-R & 5266.25 & 2450.49 \\
\hline Heart-L & 9044.33 & 6431.60 \\
\hline Heart-R & 7380.84 & 6223.27 \\
\hline Small Intestine-L & 7153.04 & 6220.43 \\
\hline Small Intestine-R & 8268.96 & 8233.55 \\
\hline Bladder-L & 6239.17 & 3131.17 \\
\hline Bladder-R & 8015.91 & 6062.63 \\
\hline Kidney-L & 5010.67 & 3657.22 \\
\hline Kidney-R & 5964.50 & 3605.58 \\
\hline Circulation-L & 13116.61 & 8812.82 \\
\hline Circulation-R & 8715.27 & 7511.83 \\
\hline Triple Heater-L & 9698.87 & 8638.97 \\
\hline Triple Heater-R & 11408.74 & 10321.59 \\
\hline Gall Bladder-L & 4048.96 & 1955.03 \\
\hline Gall Bladder-R & 4278.55 & 2344.99 \\
\hline Liver-L & 4200.96 & 3629.27 \\
\hline Liver-R & 4190.64 & 2287.51 \\
\hline
\end{tabular}

Finally, when averaged for body side, the coefficients for left side acupoints are higher than right side acupoints (averaging about a .05 difference).

\section{Discussion}

We conducted a rather technical study designed to help establish solid measurement protocols for the determination of electrical resistance at acupoints, which reflect the meridians targeted in widely practiced TCM. This was based on the reasoning that once measurement reliability is maximized, essential research on the validity and clinical meaning of acupoint activity can proceed.

The findings clearly support the measurement reliability of the Prognos device and are consistent with the reliability coefficients reported by Colbert and colleagues (Colbert et al. 2004; Pearson et al. 2007). We replicated that (1) reliability increases with repeated measurement, and (2) acupoint activity on the left side is more reliable than on the right. Further, we found that discarding the first measure did not improve reliability.

Results also indicate that one particular acupoint, the 'Right Triple Heater', displayed low reliability which could not be improved with repeated measurement. This is a finding for which we have no ready explanation. It is not likely an artifact because reliability for this one acupoint was consistently below those of other acupoints.

Overall our study revealed high reliability scores and thus replicates the observations of Colbert and her colleagues. We conclude that the Prognos is reliable in measuring electrical skin resistance at the Jing well (Ting) acupoints located at the corner of the nail bed of the fingers and toes (with the notable exception of one acupoint). To our knowledge, the Prognos device is the only device that has been tested for reproducibility by 2 separate teams of researchers and proven to be reliable in both settings. Given the general principle of improved reliability with multiple measurements (see for example blood pressure measurement; Selenta et al. 2000), we definitely suggest that readings be averaged. The strongest reliability coefficients were obtained for the average of 5 readings and we therefore recommend to average 5 readings. Interestingly, the average of four readings was not superior to that of averaging three readings and a researcher or clinician pressed for time might choose to accept the slight loss of reliability when averaging only three readings rather than five. Discarding the first reading was not supported by our data and is not recommended.

In sum, our study has provided additional foundational information about the reliability of the Prognos ohmmeter. Although our sample was relatively small, given the remarkable overlap of our findings with those of Colbert 
and her team, we feel comfortable in recommending the Prognos device in terms of measurement reliability provided the researcher does not contend himself/herself with a single reading. This conclusion also encourages further research on the clinical validation of the acupoint principle. We are curious to hear whether or not other users have had the same problem with the Right Triple heater acupoint as we had. If so, we wonder what this anomaly means in terms of the underlying principle of meridian activity.

Acknowledgments The authors wish to thank Med Prevent, Germany for loaning the Prognos Ohmmeter for use in this study and for training the experimenters in its proper use. No money was provided by MedPrevent to support the study and the authors have no financial liabilities toward this manufacturer or any product distributor. This study was entirely supported by an independent grant from the Lotte \& John Hecht Memorial Foundation, 502-325 Howe Street, Vancouver, B.C., Canada V6C 1 Z7.

Open Access This article is distributed under the terms of the Creative Commons Attribution Noncommercial License which permits any noncommercial use, distribution, and reproduction in any medium, provided the original author(s) and source are credited.

\section{References}

Ahn, A., Colbert, A., Anderson, B., Martinsen, O., Hammerschlag, R., Cina, S., et al. (2008). Electrical properties of acupuncture points and meridians: A systematic review. Bioelectromagnetics, 29, 245.

Colbert, A., Hammerschlag, R., Aickin, M., \& McNames, J. (2004). Reliability of the prognos electrodermal device for measurement of electrical skin resistance at acupuncture points. The Journal of Alternative and Complementary Medicine, 10(4), 610-616.

Colbert, A., Hayes, M., Aickin, M., \& Hammerschlag, R. (2006). Physiological variability of electrical skin resistance measurements at the ting acupuncture points. Medical Acupuncture, $17(2), 1-10$

Ezzo, J. M., Richardson, M. A., Vickers, A., Allen, C., Dibble, S. L., Isseli, B. F., et al. (2006). Acupuncture point stimulation for chemotherapy-induced nausea or vomiting. Cochrane Data Base of Systematic Reviews, (2), November 15, 2007.

Furlan, A. D., van Tulder, M. W., Cherkin, D. C., Tsukanama, H., Lao, L., Koes, B. W., et al. (1999). Acupuncture and dryneedling for low back pain. Cochrane Data Base of Systematic Reviews, (1), November 15, 2007.

Hsin, H., Huang, S. M., Chao, P. T., Jan, M. Y., Hsu, T. L., Wang, W. K., et al. (2007). Microcirculatory characteristics of acupuncture points obtained by laser doppler flowmetry. Physiological Measurement, 28, N77-N86.

Lo, S. Y. (2002). Meridians in acupuncture and infrared imaging. Journal of Medical Hypotheses, 58, 72-76.

Melchart, D., Linde, K., Berman, B., White, A., Vickers, A., Allais, G., et al. (2001). Acupuncture for idiopathic headache. Cochrane Data Base of Systematic Reviews, (1), November 15, 2007.

Pearson, S., Colbert, A. P., McNames, J., Baumgartner, M., \& Hammerschlag, R. (2007). Electrical skin impedance at acupuncture points. The Journal of Alternative and Complementary Medicine, 13(4), 409-418.

Selenta, C., Hogan, B., \& Linden, W. (2000). How often do office blood pressures fail to detect true hypertension? Archives of Family Medicine, 9, 533-540.

Szopinski, J. Z., Lochner, G. P., \& Pantanowitz, D. (2006). Influence of organ pathology on the electrical parameters of organ projection areas of the skin. Journal of Traditional Chinese Medicine, 26(3), 218-225.

Szopinski, J. Z., Pantanowitz, D., \& Jaros, G. G. (1998). South African Medical Journal, 88(2), 146-150.

Szopinski, J. Z., Pantanowitz, D., \& Lochner, G. P. (2004). Estimation of diagnostic accuracy of organ electrodermal diagnostic. South African Medical Journal, 94(7), 547-551.

Voll, R. (1978). Verification of acupuncture by means of electro acupuncture according to voll. American Journal of Acupuncture, 6(1), 1-10.

Yang, H. Q., Xie, S. S., Liu, S. H., Li, H., \& Guo, Z. Y. (2007). Differences in optical transport properties between human meridian and non-meridian. American Journal of Chinese Medicine, 35(5), 743-752.

Yung, K. T. (2004). A birdcage model for the Chinese meridian system: Part I. A channel as a transmission line. The American Journal of Chinese Medicine, 32(5), 815-828.

Zhang, W. B., Jeong, D. M., Lee, Y. H., \& Lee, M. S. (2004). Measurement of subcutaneous impedance by four-electrode method at acupoints located with single-power alternative current. The American Journal of Chinese Medicine, 32(5), 779-788. 